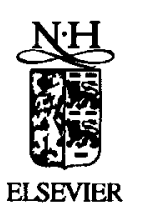

Physica A 215 (1995) 152-169

\title{
Approximations to the two-hole ground state of the Hubbard-Anderson model: a numerical test
}

\author{
M.O. Elout, M.R.M.J. Traa, W.J. Caspers \\ Center for Theoretical Physics, University of Twente, P.O. Box 217, 7500 AE Enschede, The Netherlands
}

Received 24 October

\begin{abstract}
Several resonating-valence-bond-type states are being considered as an approximation of the two-hole ground state of the two-dimensional Hubbard-Anderson model. These states have been carefully constructed by Traa and Caspers with such algebraic properties, as to optimise different contributions of the Hubbard-Anderson hamiltonian. In this paper, the different contributions to their energies are calculated for lattices with sizes from $8 \times 8$ up to $16 \times 16$ and periodic boundary conditions, using a variational Monte-Carlo method. We show which state is lowest in energy and, more important, why this is so. In accordance with the optimal state from this tested set, we propose a bound state. It will be shown that this state is indeed the most stable state.
\end{abstract}

\section{Introduction}

In the past several years, since the discovery of the high- $T_{\mathrm{c}}$ superconducting cuprates [1], there has been an increased interest in the two-dimensional Heisenberg model, and extensions thereof. Anderson [2] has proposed that the ground state of these models may contain the necessary features to describe the superconducting state in this kind of material. Following this proposition, much work has been done to gain more insight into the properties of the ground states of these models. As exact results on these two-dimensional models are scarce, the main source of information about 'exact' properties of the ground states (albeit for finite lattices) has been the results obtained from numerical simulations. One of the simplest models investigated is the $t-J$ model [3-5], which reduces to the Heisenberg model at half filling $[6,7]$.

Our group has studied the slightly more complicated Hubbard-Anderson model. We have formulated a set of two-hole states [8,9], whose phases satisfy Marshall-like relations, such as to make their energy as low as possible. Therefore, these states are believed to entail some of the algebraic properties of the true ground state of the Hubbard-Anderson model. In order to get more precise information on these states, 
we have conducted this study, in which we, by numerical simulation, determine their energies and explain why one state is more stable than others.

We will also test a state devised to be even lower in energy and to contain bound holes.

In Section 2, we will give a short introduction to the basic functions used, the form of the Hubbard-Anderson hamiltonian and define the above-mentioned states. In Section 3, we will explain the Monte-Carlo method used to obtain expectation values from the above-mentioned states on lattices ranging from $8 \times 8$ to $16 \times 16$ sites, utilizing periodic boundary conditions. In Section 4 , we will give the results obtained from the tested states and in Section 5 these data will be discussed.

\section{Theory}

\subsection{Hamiltonian}

The Hubbard-Anderson model on a square lattice is described by an effective hamiltonian which can be obtained from the large- $U$, single-band Hubbard hamiltonian [11]. Because of the large $U$, doubly occupied sites are not allowed in this model. The hamiltonian $H$ reads:

$$
\begin{aligned}
& H=H_{1}+H_{2}+H_{3}, \\
& H_{3}=H_{3,0}+H_{3,1}, \\
& H_{1}=-t \sum_{\langle i, j\rangle} \sum_{\sigma}\left(c_{i \sigma}^{+} c_{j \sigma}+\text { H.c. }\right), \\
& H_{2}=2 \frac{t^{2}}{U} \sum_{\langle i, j\rangle} \sum_{\sigma}\left(c_{i \sigma}^{+} c_{i-\sigma} c_{j-\sigma}^{+} c_{j \sigma}-n_{i \sigma} n_{j-\sigma}\right), \\
& H_{3,0}=-\frac{t^{2}}{U_{\langle i, j, k\rangle}} \sum_{\sigma}\left[\left(c_{i \sigma}^{+} c_{j \sigma} c_{j-\sigma}^{+} c_{k-\sigma}+c_{i \sigma}^{+} n_{j-\sigma} c_{k \sigma}\right)+\text { H.c. }\right], \\
& H_{3,1}=-\frac{t^{2}}{U} \sum_{\langle l, m, n\rangle} \sum_{\sigma}\left[\left(c_{l \sigma}^{+} c_{m \sigma} c_{m-\sigma}^{+} c_{n-\sigma}+c_{l \sigma}^{+} n_{m-\sigma} c_{n \sigma}\right)+\text { H.c. }\right],
\end{aligned}
$$

where $t$ is the transfer integral and $U$ the on-site Coulomb repulsion. Both $t$ and $U$ are taken to be positive, with $t \ll U$ (a physically relevant value is: $U=10 t$ [13]). A pair of nearest neighbours is denoted by $\langle i, j\rangle$, a triple of sites, $i$ and $k$ being nearest neighbours of $j$, lying all three on either one vertical or one horizontal line, is denoted by $\langle i, j, k\rangle$, and a triple of sites, $l$ and $n$ being nearest neighbours of $m$, lying at a mutual distance of $\sqrt{ } 2$ along the diagonal of one square, is denoted by $\langle l, m, n\rangle$. The $z$-component of the spin of an electron on a certain site is denoted by $\sigma\left(\sigma=\frac{1}{2}\right.$ or $\left.\sigma=-\frac{1}{2}\right)$. 


\subsection{Zero- and first-order states}

Traa et al. $[8,9]$ have given several zero-order ground states containing two holes with phases which satisfy Marshall-like relations for the operators $H_{2}$ and parts of $\mathrm{H}_{3}$.

The zero-order states are linear combinations of all possible AB hole configurations and all possible singlet-pair coverings of the spin background, such that every singlet pair is between an A and a B site. The sites of one sublattice are denoted by A, the others by $B$. If the two holes are on different sublattices we have an AB hole configuration, if they are on the same sublattice, we have an AA or BB configuration.

The amplitude of every state (i.e. a state with two holes and one singlet-pair covering on the remaining sites) in the linear combination is a product of weights, one assigned to the particular hole configuration occurring in that state and one assigned to every singlet pair occurring in that state. The singlet-pair weights are defined according to Anderson [12] using their case of a short-bond wave function with an exponential tail. The hole weights $w_{\mathrm{h}}(d)$ are as follows:

$$
w_{\mathrm{h}}(d)=\exp \left[-\operatorname{sign}(\sigma) d^{2} / \sigma^{2}\right]
$$

where $d$ is the distance between the holes measured in a Cartesian coordinate system. The parameter $\sigma$ is varied in the numerical calculations to study different hole distributions.

The phase of a zero-order state only depends on the positions of the holes, and is independent of the singlet-pair covering it contains. The phases are chosen such that either all interactions of $H_{3,0}$ and half the interactions of $H_{3,1}$ lead to negative matrix elements (the others being positive), or all interactions of $H_{3,1}$ lead to negative matrix elements (all matrix elements of $H_{3,0}$ being positive). There are several ways of dividing the group of interactions occurring in $H_{3,1}$ into two. The highest-symmetric ones are displayed in Fig. 1.

The phases are defined as follows: if one assigns to every site a sign and one lets the phase of a state be determined by the product of the signs of the two sites on which the holes reside, the phases of the four states, which either optimize $H_{3,0}$ and part of $H_{3,1}$
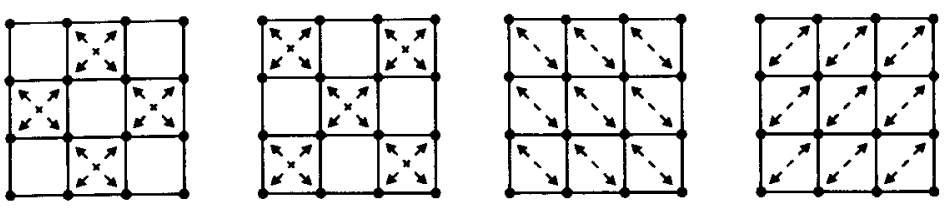

Fig. 1. The first two drawings divide the $H_{3,1}$ interactions into two groups, such that every group has the same symmetry elements as the total set of interactions, however with a doubled unit cell. The next two drawings divide the $H_{3,1}$ interactions into two groups, such that the unit cell remains the same, however with the fourfold axis reduced to a twofold axis. 

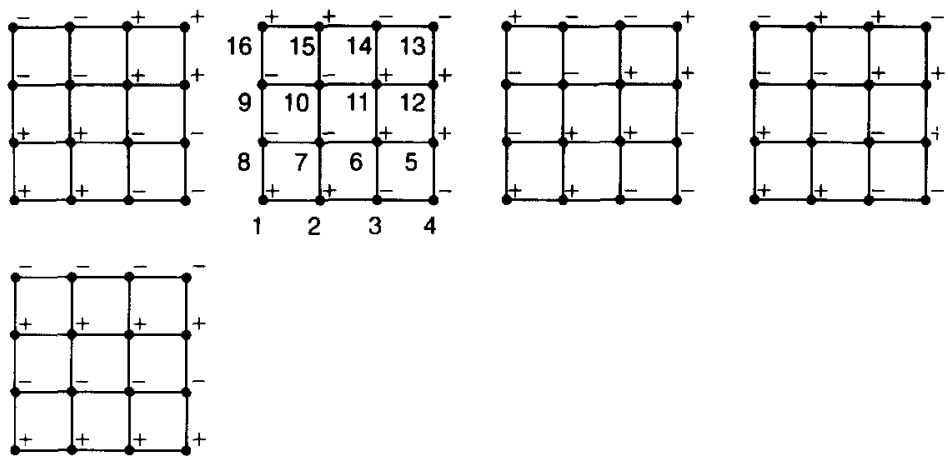

Fig. 2. The phases of the tested states are depicted. The phase of a certain hole configuration is equal to the product of the signs belonging to the sites on which the holes reside. The first four lattices represent the states optimising $H_{3,0}$ and the corresponding parts of $H_{3,1}$ given in Fig. 1. The last lattice represents the state optimising the total $H_{3.1}$ contribution.

or the total of $H_{3,1}$, can be depicted as in Fig. 2. The first four lattices in Fig. 2 represent the states optimising $H_{3,0}$ and the corresponding parts of $H_{3,1}$ given in Fig. 1. The last lattice represents the state optimising the total $H_{3,1}$ contribution.

These five states are the so-called zero-order states. They serve as a starting point to include $\mathrm{AA}$ and $\mathrm{BB}$ hole configurations. When this type of configurations is included, we arrive at the first-order states.

In these states we will allow, apart from all possible $\mathrm{AB}$ hole configurations, $\mathrm{AA}$ and BB hole configurations with the holes placed at a mutual distance of $\sqrt{2}$ along the diagonal of a square, or with the two holes separated by two steps in the $x$ - or $y$-direction from each other and zero steps in the perpendicular direction. These hole configurations necessarily contain a singlet pair between two A or two B sites. Hence, these states have a spin excitation (SE) at a certain site [9]. In the first-order states to be tested, SEs are only permitted on sites directly neighbouring both holes.

In order to have the 1-SE states optimally connected with respect to $H_{1}$ to the $0-\mathrm{SE}$ states for which one of the holes is on the SE position in the 1-SE state, we impose a further restriction according to which 1-SE states are added to the zero-order states: a 1-SE state is only added to a zero order state if the sign of the sites on which its holes reside are opposite according to the sign assignment given in Fig. 2.

The amplitudes of the 1-SE states are constructed in a manner analogous to the 0 -SE case. The singlet-pair weights of the AA or BB singlet pairs of Manhattan length $l$ are the averages of the singlet-pair weights for the singlet pairs of length $l-1$ and length $l+1$, divided by a parameter $s_{w}$. This parameter will be varied in the numerical calculations to study different relative contributions of the 1-SE states. We choose the hole weights of the 1-SE states equal to $w_{\mathrm{h}}(1)$. The phases of the 1-SE states are chosen to be optimally connected via $H_{1}$ to those 0 -SE states with one of the holes on the SE position. Traa [9] has described in more detail the first-order ground states and some of their properties. Following Traa [9], we call the investigated states 
$|1\rangle,|2\rangle,\left|K_{1}\right\rangle,\left|K_{2}\right\rangle$ and $|(0,0)\rangle$, in the ordering as given in Fig. 2. The states $|1\rangle$ and $|2\rangle$, as well as $\left|K_{1}\right\rangle$ and $\left|K_{2}\right\rangle$ are degenerate on a square lattice with periodic boundary conditions in both directions.

To summarise, we have defined five different states as a first approximation to the ground state of the Hubbard-Anderson model, with two variables, $\sigma$ and $s_{\mathrm{w}}$, to be changed in the simulations to study different hole distributions and relative contributions of the 1-SE part.

\subsection{A bound state}

As will become apparent from the numerical results obtained for the above-defined first-order states, state $|(0,0)\rangle$ will be lowest in energy. The phases of the states with neighbouring holes will be used as a starting point for defining a bound state.

We will use indices $i$ or $i_{n}$ for sites on sublattice $A$, and $j$ or $j_{m}$ for sites on sublattice B. We will now consider the genesis of first-order corrections to this starting or zero-order state.

All zero-order hole configurations (e.g. Fig. 3a and b), result in four different first-order corrections: for a 'vertical' zero-order pair the top hole as well as the bottom hole may make a shift of one elementary step to the left or to the right. Analogous processes are considered for 'horizontal' pairs with corresponding shifts in the vertical direction, either up or down. The shifts are caused by the action of $H_{1}$. The shift should always correspond with a preparatory projection on a singlet-pair state for an adjoining pair of spins.

In formula a configuration with a neighbour pair of holes, i.e. a part of the zero-order state, takes the form:

$$
|i j\rangle=C_{0}(i j)|0\rangle, \quad C_{0}(i j)=\frac{1}{2} \sqrt{2}\left(c_{j-} c_{i+}-c_{j+} c_{i-}\right)=C_{0}(j i) .
$$

The symbol $C_{0}$ indicates the creation operator for a singlet hole pair. Its hermitian conjugate represents the corresponding annihilation operator. $|0\rangle$ is a pseudovacuum state, which can be chosen to be an approximation to the ground state of $H$ in the case of half filling.

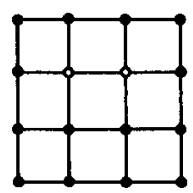

(a)

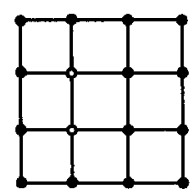

(b)

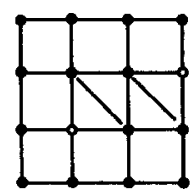

(c)

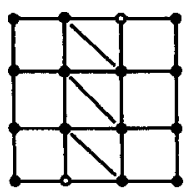

(d)

Fig. 3. Some examples of states allowed in the bound state are given. Those sites not having a hole and not being connected to a singlet pair of length $\sqrt{ } 2$ participate in a usual valence bond between an $A$ site and a B site. 
The combination of these operators in the proper order projects a general state on the part that corresponds with a singlet for the pair of electrons on positions $i j$ :

$$
P_{0}(i j)=C_{0}^{+}(i j) C_{0}(i j)=\frac{1}{2}\left(c_{i+}^{+} c_{j-}^{+}-c_{i-}^{+} c_{j+}^{+}\right)\left(c_{j-} c_{i+}-c_{j+} c_{i-}\right) .
$$

With these pair operators we can describe in a compact way the processes leading to next-nearest-neighbour hole configurations (with the holes only along the diagonal of a square). The first step results in a singlet pair adjoining the pair of holes: for the example of a vertical pair either to the left or to the right. The algebraic expression for this first step is:

$$
P_{0}\left(i_{1} j_{1}\right) C_{0}(i j)|0\rangle
$$

and the final state after the operation of the proper term of $H_{1}$ is:

$$
\sum_{\sigma} c_{j \sigma}^{+} c_{i_{1} \sigma} P_{0}\left(i_{1} j_{1}\right) C_{0}(i j)|0\rangle=C_{0}^{+}\left(j j_{1}\right) C_{0}\left(i_{1} j_{1}\right) C_{0}(i j)|0\rangle .
$$

We have written down the effect of $H_{1}$ apart from a factor $-t$, in order to give (5) and (6) the proper relative Marshall-like phase, resulting in a negative $H_{1}$ matrix element. The value of $t$ is irrelevant at this stage for the determination of (6), because, being a part of the total variational state, it has an undetermined amplitude sofar: only its complex phase (Marshall sign) relative to (5) and (3) is fixed by our procedure.

The construction resulting in (6) may be readily generalized by shifting the hole originally at $j$ one further step in the same direction after a second preliminary projection on a singlet pair. This results in states of which an example is given in Fig. 3c.

The corresponding algebraic representation of projection and shift is:

$$
P_{0}\left(i_{2} j_{2}\right) C_{0}^{+}\left(j j_{1}\right) C_{0}\left(i_{1} j_{1}\right) C_{0}(i j)|0\rangle
$$

and:

$$
C_{0}^{+}\left(i_{1} i_{2}\right) C_{0}^{+}\left(j j_{1}\right) C_{0}\left(i_{2} j_{2}\right) C_{0}\left(i_{1} j_{1}\right) C_{0}(i j)|0\rangle .
$$

In a straightforward way (8) may be further generalized to a state with e.g. an even number of parallel singlet pairs of electrons of the same sublattice.

In formula this state is represented by:

$$
C_{0}^{+}\left(i_{2 n-1} i_{2 n}\right) C_{0}^{+}\left(j_{2 n-2} j_{2 n-1}\right) \cdots C_{0}^{+}\left(j j_{1}\right) C_{0}\left(i_{2 n} j_{2 n}\right) C_{0}\left(i_{2 n-1} j_{2 n-1}\right) \cdots C_{0}(i j)|0\rangle
$$

In the series of states represented by (3), (6), (8) and (9), one of the holes originally at $j$ is step by step transported to the position $j_{2 n}$. Each step is preceded by a projection and the path the moving hole follows is parallel to the horizontal axis when the starting configuration was a vertical hole pair. An analogous reasoning holds for horizontal hole pairs. 
Now one of the essential features of configurations (9) is that the row of 'wrong' singlet pairs represented by the pair creation operators $C_{0}^{+}$may be shortened in 2 ways: either by the inverse process of the one that resulted in (9) in first instance, or by the displacement of the hole at $i$ in the direction of $i_{2 n}$, the neighbour site of the other hole at $j_{2 n}$. The first step in this last process is realized by the term

$$
\sum_{\sigma} c_{i \sigma}^{+} c_{j_{1} \sigma}
$$

of $H_{1}$, again leaving out a factor $-t$, which results in the proper Marshall sign of the final state, relative to (9). As stated before, the absolute value of the amplitude of this state should be determined by a variational procedure. It is easy to show, taking into account the proper (anti) commutation relations, that the effect of (10) on (9) gives:

$$
P_{0}(i j) C_{0}^{+}\left(i_{2 n-1} i_{2 n}\right) C_{0}^{+}\left(j_{2 n-2} j_{2 n-1}\right) \cdots C_{0}^{+}\left(i_{1} i_{2}\right) C_{0}\left(i_{2 n} j_{2 n}\right) \cdots C_{0}\left(i_{1} j_{1}\right)|0\rangle .
$$

This state has a completely analogous form to (9) apart from the projection operator $P_{0}(i j)$, which only restricts the number of Ising configurations but does not change their phases. The number of 'wrong' singlet pairs differs by one.

In this way we have constructed a set of two-hole states that are connected by matrix elements of $H_{1}$ that are all negative. The set of states originating from vertical hole pairs should have an overall minus sign, in accordance with the sign assignment given in Fig. 2. This overall minus sign is left out in the formulas (3-11).

Again, we need to define the amplitude with which each configuration will occur in this state. Every singlet pair occurring in a given configuration is assigned a weight equal to the weights used by Anderson [12] in the case of a short-bond wave function with an exponential tail. The diagonal singlet pairs (between spins on sites of the same sublattice) are given a weight of 1 .

The different hole configurations are given the weights according to equation (2).

\section{Numerical method}

We need to calculate the expectation value $\langle O\rangle$ of an operator $O$ when the system is in a state $\Psi$. This expectation value is given by:

$$
\langle O\rangle=\frac{\sum_{i} \sum_{j} w_{i} w_{j}\langle i|O| j\rangle}{\sum_{i} \sum_{j} w_{i} w_{j}\langle i \mid j\rangle},
$$

where

$$
\Psi=\sum_{i} w_{i}|i\rangle
$$


We may write

$$
\langle O\rangle=\frac{\sum_{i} \sum_{j} w_{i} w_{j}\langle i \mid j\rangle \sum_{k} \frac{\langle i|O| k\rangle}{\langle i \mid j\rangle} \frac{w_{k}}{w_{j}}}{\sum_{i} \sum_{j} w_{i} w_{j}\langle i \mid j\rangle},
$$

where the summation over $k$ is such that every matrix element $\langle i|O| k\rangle$ is included in the expectation value exactly once for the total summation over all non-zero $\langle i \mid j\rangle \mathrm{s}$.

This may be rewritten as:

$$
\langle O\rangle=\sum_{i} \sum_{j} p_{i j} \sum_{k} \frac{\langle i|O| k\rangle w_{k}}{\langle i \mid j\rangle w_{j}},
$$

where

$$
p_{i j}=\frac{w_{i} w_{j}\langle i \mid j\rangle}{\sum_{k} \sum_{l} w_{k} w_{l}\langle k \mid l\rangle} .
$$

If all $p_{i j}$ are positive, the summation in Eq. (15) can be considered as a weighted sum of $\Sigma_{k}\langle i|O| k\rangle w_{k} /\left(\langle i \mid j\rangle w_{j}\right)$ in the product space of basic functions. Hence, a MonteCarlo procedure with Metropolis sampling can be used to evaluate the expectation value. If $p_{i j}$ can also be negative, we will evaluate the quantities $\langle O\rangle^{\prime}$ and $\langle\Psi \mid \Psi\rangle^{\prime}$, defined by:

$$
\begin{aligned}
& q_{i j}=\left|w_{i} w_{j}\langle i \mid j\rangle\right|, \quad s_{i j}=\operatorname{sign}\left(w_{i} w_{j}\langle i \mid j\rangle\right), \quad q_{i j}^{\prime}=q_{i j} / \sum_{i, j} q_{i j}, \\
& \langle O\rangle^{\prime}=\sum_{i, j} q_{i j}^{\prime} s_{i j} \sum_{k} \frac{\langle i|O| k\rangle}{\langle i \mid j\rangle} \frac{w_{k}}{w_{j}}, \quad\langle\Psi \mid \Psi\rangle^{\prime}=\sum_{i, j} q_{i j}^{\prime} s_{i j} .
\end{aligned}
$$

Again, these quantities are weighted sums (with $q_{i j}^{\prime}$ being the weights), and therefore, the same Monte-Carlo method can be applied. The desired expectation value is given by:

$$
\langle O\rangle=\frac{\langle O\rangle^{\prime}}{\langle\Psi \mid \Psi\rangle^{\prime}} .
$$

The expectation values have been determined in 10 runs of 100000 Monte-Carlo steps. The values for $t$ and $U$ used are 1 and 10 . Note, however, that these are only relevant for the calculation of the total energy.

\section{Results}

Some typical results for states $|1\rangle$ and $|(0,0)\rangle$ are given in Figs. 4 and 5 . The results of $|2\rangle,\left|K_{1}\right\rangle$ and $\left|K_{2}\right\rangle$ are similar to $|1\rangle$. The set of $s_{w}$ values tested is $\{0.5,0.6, \ldots, 1.0\}$. The set of $\sigma$ values investigated is $\{1.0,2.0, \ldots, 10.0\}$. For $s_{w}=0.8$ 

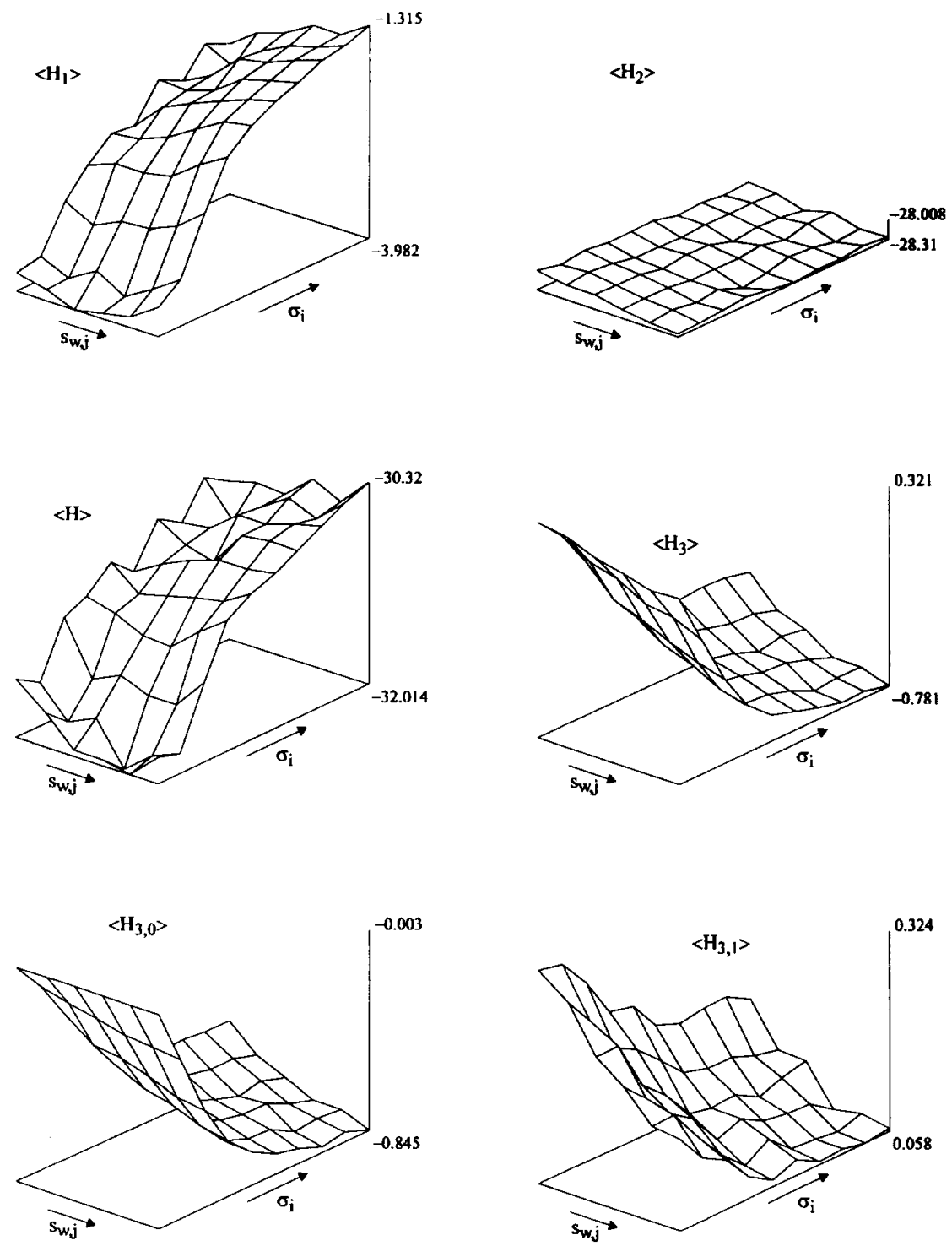

Fig. 4. Expectation values of the different components of $H$ for the state $|1\rangle$.

and $\sigma=1.0$ on an $8 \times 8$ lattice, we have calculated the different contributions in the hamiltonian more precisely, by averaging over 10 times 1000000 Monte-Carlo steps. These results can be found in Table 1. The quantity $\langle\Psi \mid \Psi\rangle^{\prime}$ in Eq. (17) in these cases has a value of the order 0.96 with a statistical error of $1 \%$.

The $\left\langle H_{1}\right\rangle$ contribution appears to be the most important factor in determining which state has the lowest energy. To get a better understanding of why the values of $\left\langle H_{1}\right\rangle$ are as they are, we have separated this quantity into several parts, every part consisting of a sum of only a specific type of matrix elements. A non-zero matrix 

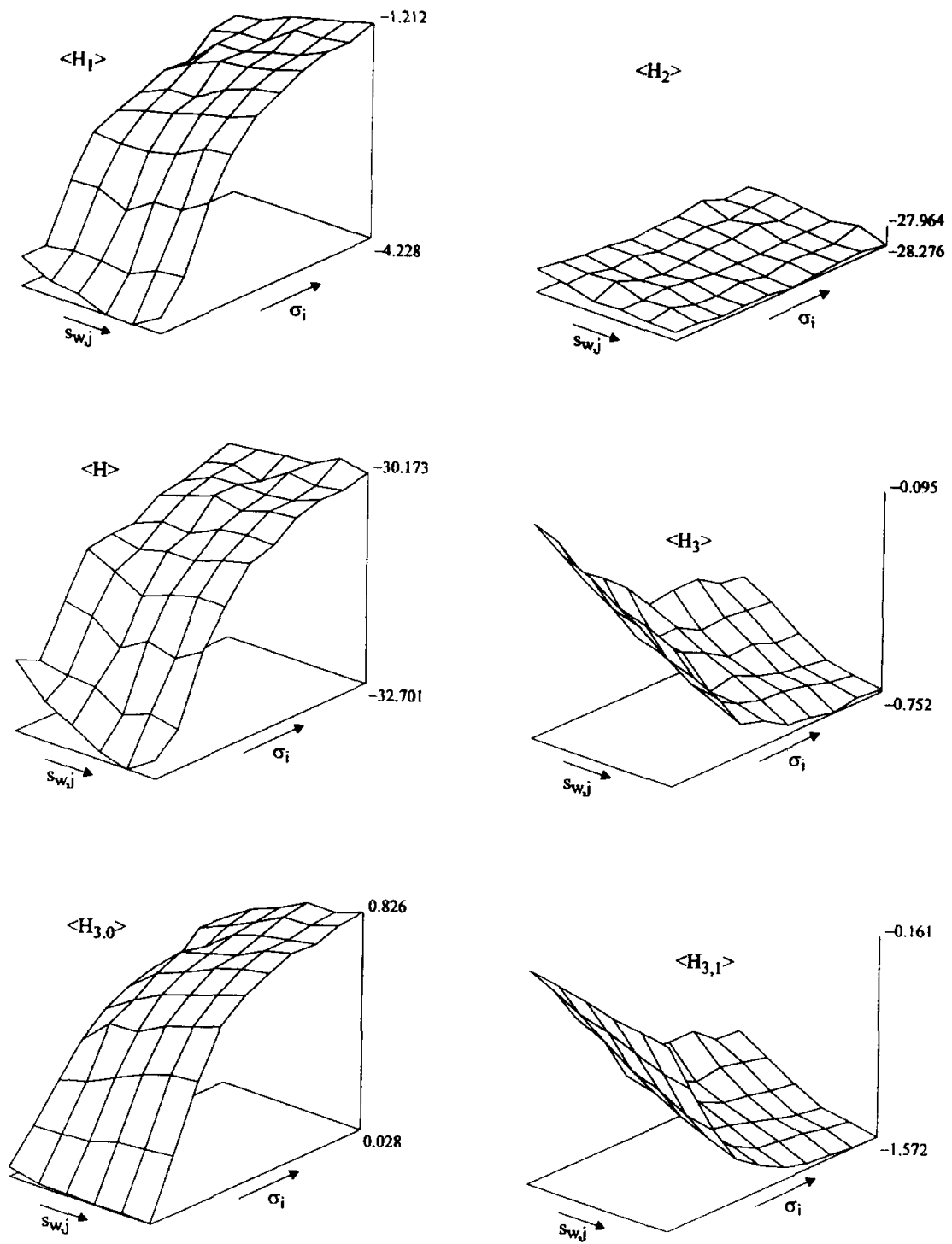

Fig. 5. Expectation values of the different components of $H$ for the state $|(0,0)\rangle$.

element $\left\langle a\left|H_{1}\right| b\right\rangle$ can be written as $c\langle d \mid e\rangle$, where $c$ is some constant, and $|d\rangle$ and $|e\rangle$ are both 1-SE functions. Either $|d\rangle$ or $|e\rangle$ is equal to $|a\rangle$ or $|b\rangle$, the other being equal to $H_{1}|a\rangle$ or $H_{1}|b\rangle$. If $|d\rangle$ and $|e\rangle$ have the same SE position, their hole positions are used as a characterisation of the matrix element to which they belong. The different possible positions are given on the second row of Table 2. If the SE positions are not identical in $|d\rangle$ and $|e\rangle$, their positions are used in the characterisation of their corresponding matrix element. 
Table 1

The expectation values of the different parts of $H$ for an $8 \times 8$ system, $s_{w}=0.8$ and $\sigma=1.0$

\begin{tabular}{|c|c|c|c|c|c|c|c|}
\hline & $\left\langle H_{1}\right\rangle$ & $\left\langle H_{2}\right\rangle$ & $\begin{array}{l}\left\langle H_{3,0}\right\rangle \\
0-\mathrm{SE}\end{array}$ & $\begin{array}{l}\left\langle\boldsymbol{H}_{3,0}\right\rangle \\
1-\mathrm{SE}\end{array}$ & $\begin{array}{l}\left\langle H_{3,1}\right\rangle \\
0-\mathrm{SE}\end{array}$ & $\begin{array}{l}\left\langle H_{3,1}\right\rangle \\
1-S E\end{array}$ & $\langle H\rangle$ \\
\hline 7 & $3.92(5)$ & $28.17(1)$ & $-0.011(2)$ & $0.00(0)$ & $0.000(1)$ & $0.216(1)$ & $-31.89(7)$ \\
\hline$|2\rangle$ & $-3.93(7)$ & $-28.16(1)$ & $-0.008(1)$ & $0.00(0)$ & $0.001(2)$ & $0.2122(8)$ & $-31.89(8)$ \\
\hline$\left|K_{1}\right\rangle$ & $-3.01(6)$ & $-28.17(1)$ & $-0.0068(7)$ & $0.00(0)$ & $-0.002(2)$ & $0.186(1)$ & $-31.00(7)$ \\
\hline$\left|K_{2}\right\rangle$ & $-3.02(4)$ & $-28.16(1)$ & $-0.0070(7)$ & $0.00(0)$ & $0.000(1)$ & $0.189(1)$ & $-31.00(5)$ \\
\hline$|(0,0)\rangle$ & $-4.19(5)$ & $-28.14(4)$ & $0.006(1)$ & $0.0263(9)$ & $-0.283(2)$ & $0.00(0)$ & $-32.59(6)$ \\
\hline
\end{tabular}

The $H_{3}$ contributions have been split into contributions from 0-SE states and 1-SE states.

Table 2

$\left\langle H_{1}\right\rangle$ split into different types of matrix elements

\begin{tabular}{|c|c|c|c|c|c|c|}
\hline $\begin{array}{l}\text { Position of the } \\
\text { holes or the SE's }\end{array}$ & $i^{0}$ & $\dot{O}^{\circ}$ & $i_{0}^{0}$ & ${ }_{0}^{0}$ & 0.0 & 0 \\
\hline $\begin{array}{l}\text { State } \| 1\rangle \\
\text { coinciding SE's } \\
\text { separated SE's }\end{array}$ & $\begin{array}{l}-0.830 \\
-0.264\end{array}$ & $\begin{array}{l}-0.827 \\
-0.256\end{array}$ & $\begin{array}{l}0.000 \\
0.003\end{array}$ & $\begin{array}{l}0.000 \\
0.004\end{array}$ & $\begin{array}{l}-0.867 \\
-0.005\end{array}$ & $\begin{array}{l}-0.874 \\
-0.008\end{array}$ \\
\hline $\begin{array}{l}\text { State }\left|K_{1}\right\rangle \\
\text { coinciding SE's } \\
\text { separated SE's }\end{array}$ & $\begin{array}{l}0.000 \\
0.269\end{array}$ & $\begin{array}{l}-0.861 \\
-0.008\end{array}$ & $\begin{array}{l}0.000 \\
0.263\end{array}$ & $\begin{array}{l}-0.859 \\
-0.001\end{array}$ & $\begin{array}{l}-0.914 \\
-0.001\end{array}$ & $\begin{array}{l}-0.894 \\
-0.005\end{array}$ \\
\hline $\begin{array}{l}\text { State }|(0,0)\rangle \\
\text { coinciding SE's } \\
\text { separated SE's }\end{array}$ & $\begin{array}{l}-0.812 \\
-0.240\end{array}$ & $\begin{array}{l}-0.802 \\
-0.243\end{array}$ & $\begin{array}{l}-0.804 \\
-0.250\end{array}$ & $\begin{array}{l}-0.804 \\
-0.250\end{array}$ & $\begin{array}{l}0.000 \\
0.000\end{array}$ & $\begin{array}{l}0.000 \\
0.000\end{array}$ \\
\hline
\end{tabular}

The $H_{1}$ contribution to the total energy (again for an $8 \times 8$ systems, $s_{w}=0.8$ and $\sigma=1.0$ ) separated into parts characterised by the 1-SE functions every matrix element implies. The SE functions are characterised by the diagrams in the second row. If the SE positions coincide, the open circles indicate the positions of the holes in that matrix element. If the SE positions do not coincide, the open circles indicate the positions of the SE's in that matrix element. An e or an o at a site indicates that the number of this site is even or odd respectively. The numbering is according to Traa et al. [9] and is given in Fig. 2.

For the bound state, we have calculated the expectation values of $H_{1}, H_{2}, H_{3}$ and all possible Manhattan distances for $8 \times 8,12 \times 12$ and $16 \times 16$ lattices with periodic boundary conditions. The results are given in the Tables 3-5 and Figs. 6-8.

\section{Discussion}

\subsection{First-order states}

To start with, we will discuss the results obtained for the first-order ground states. Let us first discuss the general forms of the surfaces given in Figs. 4 and 5. 
Table 3

The bound state for a $16 \times 16$ lattice

\begin{tabular}{lrlllllllll}
\hline$i$ & $\sigma(i)$ & \multicolumn{7}{c}{ Expectation value of Manhattan distance $m$} \\
\cline { 3 - 10 } & & $m=1$ & $m=2$ & $m=3$ & $m=4$ & $m=5$ & $m=6$ & $m=7$ & $m=8$ \\
\hline 1 & 0.5 & 0.999 & 0.001 & 0.000 & 0.000 & 0.000 & 0.000 & 0.000 & 0.000 \\
2 & 1.0 & 0.843 & 0.156 & 0.000 & 0.000 & 0.000 & 0.000 & 0.000 & 0.000 \\
3 & 1.5 & 0.642 & 0.332 & 0.026 & 0.000 & 0.000 & 0.000 & 0.000 & 0.000 \\
4 & 2.0 & 0.491 & 0.412 & 0.090 & 0.007 & 0.000 & 0.000 & 0.000 & 0.000 \\
5 & 10.0 & 0.262 & 0.341 & 0.251 & 0.087 & 0.033 & 0.016 & 0.009 & 0.002 \\
6 & -10.0 & 0.227 & 0.311 & 0.250 & 0.106 & 0.051 & 0.028 & 0.017 & 0.011 \\
7 & -5.2 & 0.120 & 0.178 & 0.171 & 0.106 & 0.078 & 0.082 & 0.105 & 0.158 \\
8 & -3.0 & 0.000 & 0.000 & 0.000 & 0.000 & 0.000 & 0.003 & 0.053 & 0.943 \\
\hline
\end{tabular}

Table 4

The bound state for a $16 \times 16$ lattice and expectation values of $H_{1}$, $\mathrm{H}_{2}$ and the total energy

\begin{tabular}{lrllll}
\hline$i$ & $\sigma(i)$ & $\left\langle H_{1}\right\rangle$ & $\left\langle H_{2}\right\rangle$ & $\left\langle H_{1}+H_{2}\right\rangle$ & $\left\langle E_{\text {tot }}\right\rangle$ \\
\hline 1 & 0.50 & $-0.5(2)$ & $-116.6(5)-117.1(7)$ & $-118.0(1)$ \\
2 & 1.00 & $-4.8(1)$ & $-117.3(1)$ & $-122.1(3)$ & $-122.7(5)$ \\
3 & 1.50 & $-6.59(9)$ & $-117.20(9)-123.8(1)$ & $-124.3(3)$ \\
4 & 2.00 & $-7.3(1)$ & $-116.77(8)-124.1(2)$ & $-124.6(3)$ \\
5 & 10.00 & $-6.83(6)$ & $-116.3(1)-123.1(2)$ & $-123.7(3)$ \\
6 & -10.00 & $-6.63(6)$ & $-116.2(1)-122.8(1)$ & $-123.3(2)$ \\
7 & -5.20 & $-5.7(1)$ & $-115.5(2)-121.2(3)$ & $-121.7(5)$ \\
8 & -3.00 & $-1.44(3)$ & $-111.9(2)-113.4(2)$ & $-113.4(2)$ \\
\hline
\end{tabular}

Table 5

The bound state for a $16 \times 16$ lattice and expectation values of $H_{3,0}$ and $H_{3,1}$

\begin{tabular}{rrrrll}
\hline$i$ & $\sigma(i)$ & $\left\langle H_{3,0}\right\rangle$ & $\left\langle H_{3,0}\right\rangle_{\text {e.d. }}$ & $\left\langle H_{3,1}\right\rangle$ & $\left\langle H_{3,1}\right\rangle_{\text {n.n. }}$ \\
\hline 1 & 0.50 & $0.000(0)$ & $0.000(0)$ & $0.000(0)$ & $-0.61(5)$ \\
2 & 1.00 & $-0.018(5)$ & $-0.015(1)$ & $0.0026(5)$ & $-0.506(6)$ \\
3 & 1.50 & $-0.17(1)$ & $-0.036(3)$ & $0.04(1)$ & $-0.388(5)$ \\
4 & 2.00 & $-0.32(2)$ & $-0.037(2)$ & $0.07(1)$ & $-0.298(5)$ \\
5 & 10.00 & $-0.48(1)$ & $-0.039(1)$ & $0.09(1)$ & $-0.165(4)$ \\
6 & -10.00 & $-0.47(2)$ & $-0.035(1)$ & $0.104(9)$ & $-0.144(5)$ \\
7 & -5.20 & $-0.50(1)$ & $-0.023(2)$ & $0.07(1)$ & $-0.074(8)$ \\
8 & -3.00 & $-0.063(2)$ & $0.000(0)$ & $0.000(0)$ & $0.000(0)$ \\
\hline
\end{tabular}

The subscript e.d. denotes 'equal distance', indicating that this column only contains contributions to $\left\langle H_{3.0}\right\rangle$ originating from hole configurations with equal mutual distance between the holes in both functions occurring in a matrix element. The subscript n.n. denotes 'nearest neighbours' and refers to matrix elements in which the holes are nearest neighbours for both states occurring in the matrix element. 

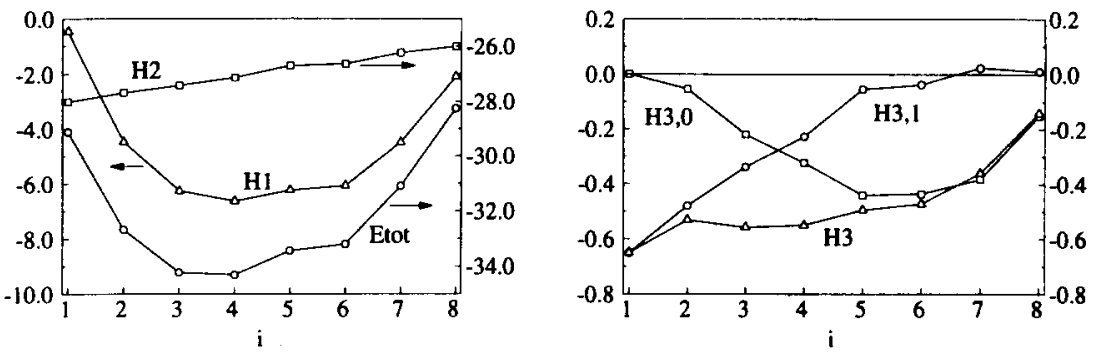

Fig. 6. Energies of the bound state for the $8 \times 8$ lattice.
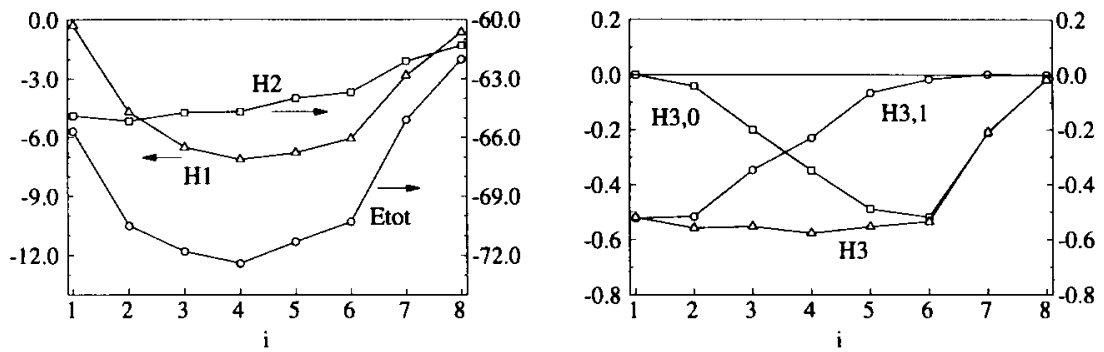

Fig. 7. Energies of the bound state for the $12 \times 12$ lattice.
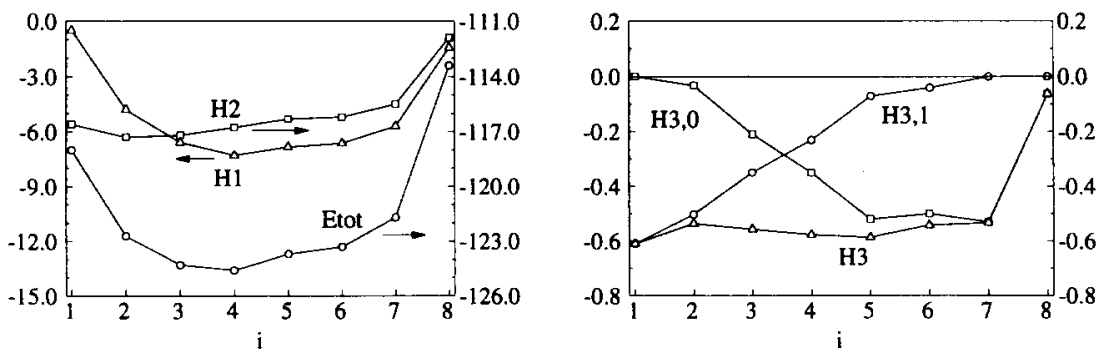

Fig. 8. Energies of the bound state for the $16 \times 16$ lattice.

$H_{1}$ is an operator which connects 0-SE states with 1-SE states. If this can be done with optimal phases, this implies that, to lower $\left\langle H_{1}\right\rangle$, one will have to include as many $0-\mathrm{SE}$ states and 1-SE states optimally connected to each other as possible. If, as described in Section 2.2, only a limited set of 1-SE states is allowed, one will find $\left\langle H_{1}\right\rangle$ to be lowest when, besides the 1-SE configurations, only those 0-SE states are included which can be optimally connected to those 1-SE states via $H_{1}$. In our case this means that for $\sigma$ a low positive number $(\sigma=1.0)\left\langle H_{1}\right\rangle$ will be lowest. For low $\sigma,\left\langle H_{1}\right\rangle$ shows a minimum around $s_{\mathrm{w}}=0.8$. For higher values of $s_{\mathrm{w}}$ the 0 -SE states become too important over the 1-SE states, and for lower values of $s_{\mathrm{w}}$ vice versa. 
$H_{2}$ is an operator which connects singlet-pair coverings, and which leaves the holes immobile. This means changing $\sigma$ does not have much effect on $\left\langle H_{2}\right\rangle$. Changing $s_{w}$, however, does, because this influences the relative weight of 1-SE states, and 1-SE states are less optimal for $\mathrm{H}_{2}$. Increasing $\mathrm{S}_{\mathrm{w}}$ will lower the weight of 1-SE states in the total state and hence will lower $\left\langle H_{2}\right\rangle$.

The operator $H_{3,0}$ connects 0 -SE states with 0-SE states, and 1-SE states with 1-SE states. First, consider the $0-\mathrm{SE}$ contribution to $\left\langle H_{3,0}\right\rangle$. The states $|1\rangle \ldots\left|K_{2}\right\rangle$ have been designed to be optimal for $H_{3,0}$, and hence their part in $\left\langle H_{3,0}\right\rangle$ is always negative. Again, as is the case for $\left\langle H_{1}\right\rangle,\left\langle H_{3,0}\right\rangle$ will be lowest if all hole configurations are connected to as many other hole configurations as possible. Hence, the 0-SE $\left\langle H_{3,0}\right\rangle$ part will be lowest for holes evenly spread throughout the lattice, i.e. for large values of $\sigma$. The state $|(0,0)\rangle$ is anti-optimal for $H_{3,0}$, and therefore, its $\left\langle H_{3,0}\right\rangle$ contribution will be minimal if its holes stay as close together as possible, i.e. for small values of $\sigma$. Now, consider the 1-SE contribution to $\left\langle H_{3,0}\right\rangle$. The states $|1\rangle \ldots\left|K_{2}\right\rangle$ all give a 1-SE contribution exactly equal to zero to $\left\langle H_{3,0}\right\rangle$. This can be most easily understood considering Fig. 2. As said previously, to optimise $H_{1}$, only those 1-SE states are allowed, for which the holes occupy sites with opposite signs. Now, because $H_{3,0}$ connects sites with opposite signs, $H_{3,0}$ operating on a $1-\mathrm{SE}$ state always leads to holes on sites with equal signs. Such states do not occur in $|1\rangle \ldots\left|K_{2}\right\rangle$, hence the 1-SE contribution equals zero. This explains why $\left\langle H_{3,0}\right\rangle$ decreases on increasing $s_{w}$ for $|1\rangle \ldots\left|K_{2}\right\rangle$. Increasing $s_{\mathrm{w}}$ increases the weight of 0 -SE states (with a negative $\left\langle H_{3,0}\right\rangle$ ) over the weight of 1-SE states (with a zero $\left\langle H_{3,0}\right\rangle$ ), leading to the found behaviour of $\left\langle H_{3,0}\right\rangle$ on $s_{\mathrm{w}}$. The $|(0,0)\rangle$ state 1 -SE contribution to $\left\langle H_{3,0}\right\rangle$ is positive. Again this can be understood from Fig. 2 . As can be seen there, $H_{3,0}$ connects sites with equal signs. This means that $H_{3,0}$ operating on a 1-SE state can lead to another 1-SE state present in $|(0,0)\rangle$, however, in general with a wrong phase, leading to a positive $\left\langle H_{3,0}\right\rangle$. This also explains the behaviour of $\left\langle H_{3,0}\right\rangle$ on $s_{w}$ for $|(0,0)\rangle$. In the case that the 1-SE states are significantly present in $|(0,0)\rangle$ (i.e. for low $\sigma$ ), reducing their contribution by increasing $s_{\mathrm{w}}$ leads to a lower $\left\langle\boldsymbol{H}_{3,0}\right\rangle$. For large $\sigma$, the holes are evenly spread throughout the lattice. In this case each 0-SE state is connected with six or eight other 0-SE states, whereas each 1-SE state is connected with four other 1-SE states in the used basis set of states. Decreasing $S_{\mathrm{w}}$ decreases the weight of 0-SE states (with many connections). As a result $\left\langle H_{3,0}\right\rangle$ decreases. As can be seen in Fig. 5, the influence of $s_{\mathrm{w}}$ on $\left\langle H_{3,0}\right\rangle$ for the state $|(0,0)\rangle$ is small.

The data on $\left\langle H_{3,1}\right\rangle$ can be explained using similar arguments as above. We will only consider the $|(0,0)\rangle$ state. The 1-SE contribution to $\left\langle H_{3,1}\right\rangle$ is exactly equal to zero. As can be seen in Fig. $2, H_{3,1}$ connects sites with opposite signs, meaning that if $H_{3,1}$ operates on a $1-S E$ function in $|(0,0)\rangle$ it will produce a $1-S E$ function not present in $|(0,0)\rangle$. The 0 -SE contribution to $\left\langle H_{3,1}\right\rangle$ is of course negative, as $|(0,0)\rangle$ has been primarily designed to be optimal for $H_{3,1}$. Increasing $\sigma$ leads to a lower $\left\langle\boldsymbol{H}_{3,1}\right\rangle$, as this leads to more evenly spread holes over the lattice, allowing more and more optimal matrix elements for $H_{3,1}$ of considerable size. Increasing $S_{\mathrm{w}}$ lowers $\left\langle H_{3,1}\right\rangle$ as this dampens the 1-SE states with zero $\left\langle H_{3,1}\right\rangle$. 
Now, we will discuss which state has the lowest energy and why this is so. We consider the case of $\sigma=1.0$ and $s_{\mathrm{w}}=0.8$, because for these values of the parameters the surfaces in Fig. 4 and 5 for the total energy appear to be close to a minimum. The relevant data are given in Table 1.

As can be seen from these data, $\left\langle H_{2}\right\rangle$ and $\left\langle H_{3,0}\right\rangle$ are approximately the same for all five states. $\left\langle H_{2}\right\rangle$ is relatively constant, because the states mainly differ in the phases chosen for different hole configurations. This property does not affect $\left\langle H_{2}\right\rangle$, as matrix elements for $\mathrm{H}_{2}$ for different hole configurations are equal to zero. $\left\langle H_{3,0}\right\rangle$ is relatively constant and almost equal to zero, as the states considered for these values of $\sigma$ and $s_{\mathrm{w}}$ contain only hole configurations with a maximum hole Manhattan distance of 2 . $H_{3,0}$ also prefers configurations with larger separations between the holes to be present.

Therefore, $\left\langle H_{1}\right\rangle$ and $\left\langle H_{3}, 1\right\rangle$ determine which states will be lowest in energy.

The reason why $\left\langle H_{3,1}\right\rangle$ favours $|(0,0)\rangle$ has in fact been given above. For these tightly bound states the 0-SE contribution to $\left\langle H_{3,1}\right\rangle$ for $|1\rangle \ldots\left|K_{2}\right\rangle$ equals zero within the error of the calculation, because these states are optimal for half of the operations present in $H_{3,1}$ and anti-optimal for the other half. The 0-SE contribution of $|(0,0)\rangle$ to $\left\langle H_{3,1}\right\rangle$ is of course optimal (i.e. negative).

The 1-SE contributions of $|1\rangle \ldots\left|K_{2}\right\rangle$ to $\left\langle H_{3,1}\right\rangle$ are positive as $H_{3,1}$ connects sites with equal signs. Therefore, in short, $H_{3,1}$ contributes up to 0.5 (on a dimensionless energy scale, for $t / U=0.1)$ to the stability of $|(0,0)\rangle$ over the other four states.

The other approximately equally important contribution to the stability of $|(0,0)\rangle$ is of course $H_{1}$. The appropriate data are given in Table 2. In this table the data for $|2\rangle$ and $\left|K_{2}\right\rangle$ have been omitted, as these are very similar to the data of $|1\rangle$ and $\left|K_{1}\right\rangle$ respectively.

First, consider the rows in Table 2, referred to as coinciding SE's. As explained in Section 4 , these rows contain the contributions to $\left\langle H_{1}\right\rangle$ for which one of the states in the matrix element has a hole on the position where the other state in the matrix element has an SE (the other holes of course residing on the same site). As these matrix elements were to be optimised in choosing the phases of the 1-SE states relative to the 0 -SE states, it should not be surprising that all entries in these rows are either zero or negative. Those entries are zero belonging to a 1-SE state (as specified by the diagrams in the second row of the table), which does not appear at all in the proposed ground state under consideration. The sum of all these types of contributions does not vary too much for the different states, and the main reason for the stabilisation of $|(0,0)\rangle$ by $H_{1}$ can be found in the rows referred to as separated SE's in Table 2.

Let us write a matrix element contributing to the case of separated SE's as $\left\langle a\left|H_{1}\right| b\right\rangle$. Furthermore, assume that $|a\rangle$ is a $1-\mathrm{SE}$ function and that $|b\rangle$ is a $0-\mathrm{SE}$ function, both being one certain singlet-pair covering each. If we write $|d\rangle=H_{1}|b\rangle$, then we have that $\left\langle a\left|H_{1}\right| b\right\rangle=c\langle a \mid d\rangle$, where $c$ is some constant. In the case of separated SE's we have that the SE's in $|a\rangle$ and in $|d\rangle$ do not reside on the same position, but on the positions referred to by the open circles in the diagrams on the second row of Table 2 . 
Those entries in a separated-SE row, which are (almost) zero, either require $|a\rangle$ to be a 1-SE state not present in the studied ground state, or require $|b\rangle$ to be a 0-SE state in which the holes are separated over a Manhattan distance equal to 3, which also almost never occurs in the tested states ( $\sigma$ being equal to 1.0). The other way around one can say, those non-zero entries originate from matrix elements for which $|a\rangle$ is an allowed 1-SE function and for which $|b\rangle$ is a 0-SE function with neighbouring holes.

What is important now, is to understand why the signs of the non-zero entries in the separated-SE case are as they are. Define $\left|a^{\prime}\right\rangle$ as the 0-SE state from which $|a\rangle$ derived its phase, i.e. $\left|a^{\prime}\right\rangle$ has one of its holes on the SE position in $|a\rangle$. This can be done in 2 ways, so also choose one of the holes of $\left|a^{\prime}\right\rangle$ on the same position as one of the holes in $|b\rangle$. The phase of $|a\rangle$ is given as $-s_{a^{\prime}} p_{a^{\prime}}$, where $s_{a^{\prime}}$ is the real phase $H_{1}$ produces when operating on $\left|a^{\prime}\right\rangle$ to give $|a\rangle$, and $p_{a^{\prime}}$ is the original phase of $\left|a^{\prime}\right\rangle$ in the chosen zero-order state. The extra minus sign is added such that $|a\rangle$ is optimally connected to $\left|a^{\prime}\right\rangle$ with respect to $H_{1}$. At this point we also define $p_{b}$ to be the phase of $|b\rangle$ and $s_{b}$ to be the real phase $H_{1}$ produces when operating on $|b\rangle$ to give $|d\rangle$. The phases $s_{a^{\prime}}$ and $s_{b}$ are equal to each other, because the hole positions in $|a\rangle$ and $|d\rangle$ are the same and because $\left|a^{\prime}\right\rangle$ and $|b\rangle$ have one hole position in common.

If one determines the phases of $\left|a^{\prime}\right\rangle$ and $|b\rangle$ from Fig. 2 for all different non-zero entries in the separated-SE case in Table 2, one finds that these entries are negative (i.e. optimal) if those phases are opposite to each other, and that they are positive if those phases are equal to each other. With $c$ (from $c\langle a \mid d\rangle$ ) being $-s_{a} \cdot p_{a^{\prime}} s_{b} p_{b}$, we find that, if the phases are opposite, $c$ will be positive, and if the phases are equal, $c$ will be negative. This means that on the average $\langle a \mid d\rangle$ will be negative, if in $|a\rangle$ and $|d\rangle$ the SE's do not reside on the same position. This is not surprising, because all overlaps in $\langle a \mid d\rangle$ between singlet-pair coverings in which one of the singlet pairs connects both SE sites are always negative, whereas the other overlaps lead to larger loops in the loopcovering and partially cancel each other, probably leading to a smaller contribution to the total value of $\langle a \mid d\rangle$. So, one may state that if the phases of $\left|a^{\prime}\right\rangle$ and $|b\rangle$ are opposite, their separated-SE contribution to $\left\langle H_{1}\right\rangle$ is optimal, and if they are equal, it is anti-optimal. Instead of considering the phases of $\left|a^{\prime}\right\rangle$ and $|b\rangle$, one may also consider the phases of $|a\rangle$ and $|d\rangle$, being the two 1-SE functions with the holes on the same position, but with the SE's on different positions. If the phases of $\left|a^{\prime}\right\rangle$ and $|b\rangle$ are opposite, then so are the phases of $|a\rangle$ and $|d\rangle$, and likewise for equal phases. Hence, one can rephrase the above: if the phases of two 1-SE functions with equal hole but different SE positions are opposite they can be associated to a negative contribution to $\left\langle H_{1}\right\rangle$ and to a positive contribution if their phases are equal.

This explains all the data in Table 2 and why $|(0,0)\rangle$ is the most stable state. State $|1\rangle$ and $|2\rangle$ have two types of 1-SE hole positions which allow for 2 different SE positions (e.g. for state $|1\rangle$ the diagonal hole positions on even squares) leading to an optimal contribution to $\left\langle H_{1}\right\rangle$. State $\left|K_{1}\right\rangle$ and $\left|K_{2}\right\rangle$ have two types of 1-SE hole positions which allow for 2 different SE positions (e.g. for state $\left|K_{1}\right\rangle$ the hole positions on a diagonal, going from bottom right to top left) leading to an anti-optimal 
contribution to $\left\langle H_{1}\right\rangle$. Finally, state $|(0,0)\rangle$ has four types of 1-SE hole positions allowing for two different SE positions (all diagonal hole positions) leading to an optimal contribution to $\left\langle H_{1}\right\rangle$.

If one wants to extend the ground state by including more AA and BB states, such as to lower $\left\langle H_{1}\right\rangle$, it is clear that this must be done by using the correct phases for the different SE positions, i.e. one should use $|(0,0)\rangle$ as a starting point. This has in fact been done in the bound state discussed below, where furthermore the number of singlet-pair coverings has been restricted to make all matrix elements of $H_{1}$ optimal.

\subsection{The bound state}

We will now discuss the results obtained for this bound state. First consider the probabilities of finding the holes at a certain Manhattan distance for a given $\sigma$. Two effects have an influence on the distribution of the holes. According to Eq. (2) a positive small $\sigma$ will lead to bound holes, whereas a small negative $\sigma$ will lead to states in which the holes tend to be separated as much as possible. This behaviour can easily be recognised in the table containing the Manhattan data. However, if $\sigma$ tends to large values, the hole weight, as given in Eq. (2), becomes independent of the Manhattan distance between the holes. Still, as can be seen in the $16 \times 16$ data for $i=6$, the probability to find the holes close together is much larger than the probability to find them separated. This is due to the fact that for the holes close together more singlet-pair coverings are allowed. This means that states with holes close together have a larger contribution in the considered state. The effect of both contributions to the probability distribution of the holes can be seen for $i=7$ for the $16 \times 16$ lattice, where two maxima occur, one for the holes close together and one for the holes separated maximally.

The dependence of $\left\langle H_{1}\right\rangle$ and $\left\langle H_{2}\right\rangle$ on the calculated hole distribution is fairly straightforward. $\left\langle H_{1}\right\rangle$ tends to be more optimal if the holes are more evenly spread over the lattice, with a slight preference for the holes on the diagonal of a square, because this state has $8 \mathrm{H}_{1}$ matrix elements whereas most other states have only 4 matrix elements. $\left\langle H_{2}\right\rangle$ favours a bound state, because, when the holes are close together, the number of singlet pairs along a diagonal of a square, which are less optimal for $\left\langle\mathrm{H}_{2}\right\rangle$, is small. Furthermore, the number of possible singlet-pair coverings is larger for bound holes, hence increasing the resonance, resulting in a lower $\left\langle\mathrm{H}_{2}\right\rangle$.

$\left\langle H_{3,0}\right\rangle$ and $\left\langle H_{3,1}\right\rangle$ actually have little effect on the binding of the holes. $\left\langle H_{3,0}\right\rangle$ prefers the holes to be fairly evenly distributed over the lattice, connecting as many different hole configurations as possible. $\left\langle H_{3,1}\right\rangle$ prefers the holes to be neighbours, as these states have optimal phases to each other with respect to $H_{3,1}$. These two expectation values combined give a contribution to the energy which is relatively insensitive to the distribution of the holes, as is clear from the Figs. 6-8.

Hence, combining all expectation values in the total energy leads to a state with lowest energy for which the probability to find holes at a Manhattan distance of less than 4 is more than $99 \%(16 \times 16$ lattice, $i=4)$. 
It might seem to the reader that this result could very well be caused by the choice of the specific bound state, and that it might not be a property of the true ground state. However, our data are supported by recent Green's-function Monte-Carlo studies [5]. In [5] the true ground state of the $t-J$ model (i.e. $H_{1}+H_{2}$ ) for $J=0.4$ (i.e. $U=10 t$ ) is determined in an 'iterative' manner, starting from a suitable trial function. In principle the applied method in [5] should produce the properties of the exact ground state. For an $8 \times 8$ lattice, the calculated two-hole distribution function shows that the probability of finding two holes along one horizontal or vertical line at a mutual distance equal to 2 or 3 or at a mutual distance of $\sqrt{8}$ along the diagonal of two squares is much lower than for other separated hole distributions, with mutual distances $\sqrt{ } 2, \sqrt{5}$ and $\sqrt{ } 10$. This somewhat surprising result is in perfect agreement with the above proposed bound state.

\section{Conclusions}

We have calculated the energies of several first-order ground states and we have shown that the state with optimal phases for $H_{3,1}$ is the most stable one. We have shown that this is so because of a favourable $\left\langle H_{3,1}\right\rangle$ and because the phases of the 1-SE configurations are correct to give an optimal contribution to $\left\langle H_{1}\right\rangle$ via matrix elements with $H_{1}$ in which one of the holes does not move to an SE position already present in the matrix element.

Furthermore we have investigated a bound state, optimising all phases for $H_{1}$, starting with $H_{3,1}$ phases for neighbouring holes. This state is lowest in energy and favours binding of the holes.

\section{References}

[1] J.B. Bednorz and K.A. Müller, Z. Phys. B 64 (1986) 189.

[2] P.W. Anderson, Science 235 (1987) 1196.

[3] T. Barnes, A.E. Jacobs, M.D. Kovarik and W.G. Macready, Phys. Rev. B 45 (1992) 256.

[4] M. Boninsegni and E. Manousakis, Phys. Rev. B 46 (1992) 560.

[5] M. Boninsegni and E. Manousakis, Phys. Rev. B 47 (1993) 11897.

[6] N. Trivedi and D.M. Ceperly, Phys. Rev. B 41 (1990) 4552.

[7] R.A. Sauerwein and M.J. de Oliveira, Phys. Rev. B 49 (1994) 5983.

[8] M.R.M.J. Traa and W.J. Caspers, Physica A 183 (1992) 175.

[9] M.R.M.J. Traa, W.J. Caspers and E.J. Banning, Physica A 203 (1994) 145.

[11] A.H. MacDonald et al. Phys. Rev. B 37 (1988) 9753;

J.E. Hirsch, Phys. Rev. Lett. 54 (1985) 1317.

[12] S. Liang, B. Doucot and P.W. Anderson, Phys. Rev. Lett. 61 (1988) 365.

[13] E. Dagotto et al. Phys. Rev. B 45 (1992) 10741. 